\title{
RESEARCH
}

\section{Pulmonary and overall healthcare utilization after childhood and young adult thyroid cancer}

\author{
Giancarlo Di Giuseppe 1,2, Aleksandra M Zuk1,3, Jonathan D Wasserman ${ }^{4,5}$ and Jason D Pole1,2,6 \\ 1Pediatric Oncology Group of Ontario, Toronto, Ontario, Canada \\ 2Dalla Lana School of Public Health, University of Toronto, Toronto, Ontario, Canada \\ ${ }^{3}$ Health Studies, and the Department of Physical and Environmental Sciences, University of Toronto Scarborough, Toronto, Ontario, Canada \\ ${ }^{4}$ Division of Endocrinology, The Hospital for Sick Children, Toronto, Ontario, Canada \\ ${ }^{5}$ Department of Pediatrics, University of Toronto, Toronto, Ontario, Canada \\ ${ }^{6}$ Centre for Health Services Research, The University of Queensland, Brisbane, Queensland, Australia
}

Correspondence should be addressed to G Di Giuseppe: gdigiuseppe@pogo.ca

\begin{abstract}
Differentiated thyroid carcinoma (DTC) in children, adolescents, and young adults is associated with excellent prognosis. However, little is known about the long-term utilization of healthcare resources among survivors. The objective of this study was to compare the utilization of healthcare resources among DTC survivors to the general population, with a focus on pulmonary-related resource utilization. Population-based linked data were used to retrospectively ascertain DTC patients from the Ontario Cancer Registry, age <30 years at diagnosis between 1992 and 2011. DTC cases were individually matched 1:10 to the general population on age, sex, diagnosis year, and rural status.

Billing records for all-cause hospitalizations, emergency department visits, pulmonary subspecialist visits, and chest imaging were compared using incidence rate ratios (IRR). A total of 2456 individuals with DTC were identified, with women representing the majority (82.8\%) of cases. Compared to controls, hospitalization and emergency department visit IRRs were $2.56(95 \% \mathrm{Cl}, 2.49-2.63)$ and 2.22 (95\% Cl, 2.18-2.26), respectively. An excess of pulmonary diagnostic imaging was observed for chest radiography (IRR, 2.7;

$95 \% \mathrm{Cl}, 2.6-2.8$ ) and pulmonary CT's (IRR, 17.5; $95 \% \mathrm{Cl}, 15.8-19.3)$. Patients who underwent radioactive iodine therapy had a higher incidence of pulmonary-related healthcare utilization than those who did not. The highest healthcare utilization for all modalities was observed in the 30 to 90 days after DTC diagnosis with a steady decline further from diagnosis; however, elevated utilization was observed throughout the observation period. Health services utilization is high for primary DTC survivors, irrespective of the time since diagnosis. Radioactive iodine therapy is associated with higher rates of utilization. These findings have ramifications for treatment, surveillance and for policy formulation.
\end{abstract}

\author{
Key Words \\ - thyroid carcinoma \\ - health services utilization \\ - medical imaging \\ - cancer survivors \\ - pediatric \\ - adolescent and young adult
}

Endocrine-Related Cancer (2020) 27, 391-402

\section{Introduction}

Differentiated thyroid carcinoma (DTC) is the most common endocrine malignancy, and the most frequently diagnosed malignancy among adolescents and young adults (Araque et al. 2017), with a significant female predominance (Massimino et al. 2018). The incidence of DTC in North America is rising (Vergamini et al. 2014, Pole et al. 2017), although the prognosis is highly favorable, with 5-, 15- and 30-year disease-specific survival 
of nearly $100 \%$ among those $0-20$ years at diagnosis (Golpanian et al. 2016). As a consequence, there is a large, and growing 'at-risk' population of adolescent and young adult survivors treated for DTC, with potential medical sequelae of their disease or treatment-related adverse effects (Araque et al. 2017).

Treatment of DTC is primarily surgical. Historically, a focus on attaining a disease-free state prompted widespread utilization of adjuvant radioactive iodine (RAI) therapy. In 2015, clinical practice guidelines for treatment of children with DTC prompted a transition to more selective utilization of RAI for those with intermediate to high risk of persistent or recurrent disease (Francis et al. 2015). RAI is generally recognized to be safe, although recent evidence suggests potential for clinical sequelae with prolonged latency, including salivary gland dysfunction, second primary malignancies and pulmonary fibrosis, particularly among those with diffuse pulmonary metastases (Marti et al. 2015, Zhang et al. 2016, Albano et al. 2017). Life-long follow-up for recurrent disease is advocated (Francis et al. 2015), although the optimal frequency and modality of surveillance has not been clearly defined.

Pulmonary metastases are common among young patients, with an estimated prevalence of 20-27\% (Bal et al. 2004, Golpanian et al. 2016, Lazar et al. 2016, Livhits et al. 2016, Alzahrani et al. 2019), in contrast to $5 \%$ of adults with DTC (Chopra et al. 2015, Lim et al. 2017). Even in the face of distally metastatic disease, mortality remains low in young patients (Pawelczak et al. 2010); however, only a minority of patients clear their disease completely (Durante et al. 2006, Song et al. 2015, Alzahrani et al. 2019). The long-term health consequences of distally metastatic disease among survivors, and impacts on healthcare utilization, have not been well defined.

The province of Ontario, Canada is a single-payer healthcare system. All encounters with the healthcare system are billed centrally, allowing the entire population to be captured using linked administrative healthcare databases. We sought to determine whether children, adolescents and young adult (CAYA) survivors of DTC, age 0-29 years at diagnosis, were accessing the healthcare system more frequently than the general population. Using provincial administrative databases, we identified CAYA diagnosed with DTC and assessed how frequently they were accessing the healthcare system. Given the relatively high rate of pulmonary metastases within the CAYA population, we elected to focus on pulmonary-related utilization. We then compared this with a matched sample of the general population with no medical history of cancer.

\section{Materials and methods}

\section{Data sources, study population and cohort creation}

A population-based retrospective matched cohort study was performed using the Ontario Cancer Data Linkage ('cd-link') project, an initiative which has been previously described (Earle 2014). In brief, a unique encrypted health insurance number was used to deterministically link administrative healthcare databases at ICES for every individual eligible for health insurance coverage in the province of Ontario, Canada. The Ontario Cancer Registry (OCR), a population-based provincial cancer registry of all incident cancer diagnoses, was used to identify thyroid carcinoma cases. Hospitalization data were obtained from the Canadian Institute for Health Information (CIHI) Discharge Abstract Database (DAD), which contains information from the discharge abstracts of all Ontario hospital admissions starting in 1988. Beginning in 2002, emergency department (ED) visits for all hospital- and community-based ambulatory care were identified from CIHI using the National Ambulatory Care Reporting System (NACRS) database. The Ontario Health Insurance Plan (OHIP) database contains all fee-forservice physician billing records and was used to identify interactions with the healthcare system (including ED visits prior to 2002) classified according to billing codes established by the provincial health ministry (Ontario Ministry of Health and Long Term Care 2015).

Establishment of this cohort was previously described (Pole et al. 2017). Briefly, thyroid carcinoma diagnoses were identified using International Classification of Disease, 9th edition (ICD-9) code 193 for malignant neoplasm of the thyroid gland. Primary thyroid cancer cases were identified in CAYA subjects aged $<30$ years with a diagnosis in the OCR between January 1, 1992 and December 31, 2011. To ensure the highest level of diagnostic certainty, only individuals whose cancer case status was ascertained by the Regional Cancer Centre Registration or by a confirmed pathology report were included. Individuals with a previous cancer prior to the incident thyroid carcinoma $(n=44)$ were excluded. Medullary thyroid carcinoma cases $(n=47)$ were identified using ICD-O histology codes (World Health Organization 2013): 8510, 8511, 8246, and 8345 and excluded from the cohort due to the association of this cancer subtype's association with hereditary cancer syndromes which could confound future healthcare utilization (Kloos et al. 2009).

DTC cases were followed from the diagnosis date (index date) of their thyroid carcinoma and censored at 
the earlier of a secondary malignant neoplasm, death, or date of last contact with the healthcare system. A reference population with no previous medical history of cancer was identified using a random sample of the Ontario population during this study period. Cases were individually matched 1:10 to controls on age, sex, rural status, and diagnosis index year using an exact greedy match (Bergstralh et al. 1996). Controls were followed from the same index date as their matched case and were censored at a cancer diagnosis or death or end of study follow-up. All matched controls were censored when a case was censored. Ethics approval was obtained from The Hospital for Sick Children, Toronto, Ontario, Canada.

\section{Pulmonary healthcare utilization and other covariates}

Administrative databases (CIHI-DAD, OHIP and NACRS) were used to identify pulmonary-related health service utilization (HSU) beginning 30 days after the diagnosis date (index date) until December 31, 2011. A start date of 30 days after the thyroid cancer diagnosis was chosen to capture utilization subsequent to early clinical therapies, as the majority of definitive surgeries would have been completed by this time. Chest radiography (CXR) and computerized tomography (CT) were identified using hospital and out-patient procedure billing codes. The Canadian Classification of Diagnostic, Therapeutic, and Surgical Procedures and the Canadian Classification of Health Interventions coding structure were used to identify CXR and CT utilization (Supplementary Table 1 , see section on supplementary materials given at the end of this article) (Statistics Canada 1986, Canadian Institute for Health Information 2012b). Pulmonary function tests (PFTs) were captured from the OHIP database for the most common test types: simple spirometry, flow-volume loop, plethysmography, carbon monoxide diffusing capacity, and oxygen saturation (Gershon et al. 2012). A full list of the pulmonaryrelated utilization procedure codes used are supplied in Supplementary Table 1. DTC patients that underwent RAI therapy after the diagnosis date of DTC (index date) were identified using the OHIP billing code X326. Imaging procedure codes for pulmonary-related HSU were captured across the CIHI-DAD, NACRS, and OHIP databases and were de-duplicated using the billing date associated with the imaging study. This de-duplication procedure was performed: (i) to prevent overestimating utilization; and (ii) under the assumption that survivors would infrequently undergo multiple pulmonary-related imaging studies late into survivorship on the same day.
Incident hospital admissions and ED visits for all causes were captured during the observation period. The primary diagnostic code associated with each hospitalization and/or ED visit was obtained using ICD-9-CM and ICD10-CA codes (US Department of Health and Human Services 1988, Canadian Institute for Health Information 2012a). Diagnostic codes were converted between ICD-9 and ICD-10 using a conversion file maintained by ICES. Fee-for-service billing records were analyzed from OHIP to identify the responsible physicians' area of specialty. This is a list of specialties or disciplines recognized by the Royal College of Physicians and Surgeons of Canada relevant to services covered under the Ministry of Health and Long-Term Care. Physician subspecialty was categorized as general practitioner, respiratory diseases specialist, otolaryngologist, or endocrinologist based on the physicians' declared specialty associated with the billing record (Ontario Ministry of Health and Long Term Care 2015).

Sociodemographic characteristics of the population were determined from administrative records. Age, sex, and index year were captured at the index date of cohort entry. Area based neighborhood income quintiles were determined by linking Canadian Census data at the index date using the residential postal code, using methods that have been described previously (Pole et al. 2017). Urban and rural status were also determined based on residential postal code at index date.

Sensitivity analysis was performed ad hoc to determine HSU for other anatomical sites throughout the study period. Abdominal and pelvic CT exams were selected as a 'negative tracer' (Supplementary Fig. 1), as the abdomen and pelvic regions are unlikely to be involved in the direct context of either primary thyroid carcinoma or with its associated therapies. In this context the 'negative tracer' is unlikely to be impacted by the underlying diagnosis or intervention (i.e. DTC treatment in this study), and thus remains constant over the period of investigation. It therefore serves as a control to demonstrate a true excess of utilization in the measure of interest and temporal trends, rather than a generalized increase in utilization of the healthcare system or of specific diagnostic modalities.

\section{Statistical analysis}

Frequencies and proportions were calculated to describe population characteristics and were stratified by sex. Nonparametric and $\chi^{2}$ tests were used to compare differences in the distributions of baseline characteristics among cases 
and controls. Incidence rates for HSU were calculated overall, and for the following time periods after diagnosis (index date): 30 to $<90$ days, 90 to $<180$ days, 180 days to $<1$ year, 1 to $<2$ years, 2 to $<3$ years, 3 to $<5$ years, 5 to $<10$ years, 10 to $<15$ years, and 15 to 21 years. These groupings were chosen to capture any changes in utilization rates during active treatment for thyroid cancer immediately after diagnosis and long into the survivorship period. Rates were calculated as events per 1000 person-years of observation and incidence rate ratios (IRRs) were calculated comparing DTC cases with controls. Confidence intervals $(95 \% \mathrm{CI})$ for the IRR were calculated using the Poisson distribution approximation. Where appropriate, analyses were stratified by the inclusion of RAI therapy as part of the treatment protocol to determine differences in HSU among DTC patients. Reported $P$ values represent two-sided tests with values $<0.05$ considered statistically significant. Statistical analyses were performed in SAS version 9.4 (SAS Institute) at the Pediatric Oncology Group of Ontario.

\section{Results}

A total of 2456 CAYA patients were diagnosed with DTC during the study period and matched to 24,560 population controls. Both cases and controls had a median and interquartile range (IQR) follow-up time of 7.4 years (IQR, 3.8-12.2). Characteristics of the cohort stratified by sex are presented in Table 1. Overall, 2034 $(82.8 \%)$ of diagnoses were identified in women. AYAs aged 20-29 years represented most incident thyroid cancer patients in this cohort $(n=2031 ; 82.7 \%)$. Approximately $8 \%$ of DTC patients were individuals living in a rural area. No difference in mortality was observed for DTC patients $(n=9,0.4 \%)$ when compared to controls $(n=115,0.5 \%)$.

Slightly more than half $(n=1390,56.6 \%)$ of the DTC patients underwent RAI therapy. Among DTC patients there was no impact of sex on rates of RAI therapy, CXR, chest CT, PFTs, or respiratory disease subspecialty billing (all $P>0.05$ ).

\section{Hospitalizations, emergency department visits and diagnoses associated with hospital visits}

All-cause hospitalization and ED visit incidence (beginning 30 days after the index date) are illustrated in Fig. 1. Overall, the IRR of all-cause hospitalizations for DTC patients was 2.56 times larger relative to the general population (95\% CI, 2.49-2.63). Similarly, ED visits had an
IRR 2.22 (95\% CI, 2.18-2.26) times larger than population controls. The highest incidence in hospitalizations and ED visits were seen in the time-period closest to the index date and found to decrease over time (Fig. 1A). There was a late increase in hospitalizations and $\mathrm{ED}$ visits in the 15- to 21-year period after primary treatment relative to the immediately preceding time periods (Fig. 1A). When stratified by RAI therapy, individuals who underwent RAI were associated with higher IRRs of hospitalizations and ED visits than those who did not undergo RAI (Fig. 1B). The overall IRR for individual in-patient hospitalization of patients who underwent RAI therapy was 2.93 (95\% CI, 2.84-3.03) versus 2.10 (95\% CI, 2.01-2.19) for those who did not receive RAI. Similarly, total ED visits were higher in the RAI group (IRR, 2.50; 95\% CI, 2.30-2.41) than those who did not receive RAI (IRR, 2.03; 95\% CI, 1.96-2.09). Absolute incidence rates for hospitalizations and ED visits stratified by time period are presented in Supplementary Table 2 .

The primary indications for hospitalization in DTC patients were identified as 'neoplasm' (35.2\%) related to the primary DTC, pregnancy and childbirth $(18.6 \%)$, health services for medical procedures and aftercare (14.5\%), digestive system diseases (6.7\%), and genitourinary diseases (5.4\%). Hospitalizations for unaffected controls followed a similar pattern; pregnancy and childbirth (51.3\%), genitourinary diseases (11.0\%), digestive system diseases (10.3\%), and symptoms and illdefined conditions (3.3\%). When stratified by time since index, pregnancy and childbirth, genitourinary diseases, and digestive system diseases were frequently among the top four diagnostic reasons of hospitalization for both DTC cases and controls two years post index date (Supplementary Table 3).

\section{Incidence and incidence rate ratio of pulmonary-related health services utilization}

There was a significant excess of individuals with DTC accessing pulmonary-related health services beginning 30 days after the index date when compared with the general population. Specifically, there was increased use of CXR (57.5 vs $28.5 \%$ ), chest CT (21.6 vs $1.7 \%$ ), PFT (15.7 vs $10.5 \%)$, and consultation with a respiratory disease specialist (6.7 vs $3.0 \%$ ) when compared to controls (all $P<0.001)$.

Time-period-specific and overall incidence rates and IRRs for pulmonary-related HSU are presented in Fig. 2 and Table 2. Overall, the IRR of CXR was 2.7 (95\% CI, 2.6-2.8), while the incidence rates for chest https://erc bioscientifica.com

https://doi.org/10.1530/ERC-19-0463 (c) 2020 Society for Endocrinology Published by Bioscientifica Ltd. Printed in Great Britain 


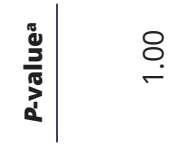

$\stackrel{8}{\circ}$

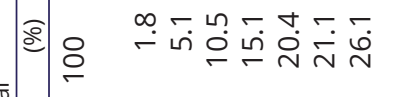

$\underset{m}{\infty} \cdot \vec{m} \dot{m}$

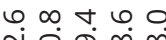

ล่ํำำ

$\stackrel{8}{\circ}$

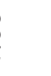

용

$\dot{z} \mid \frac{8}{2}$

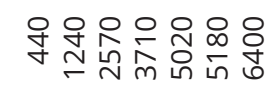

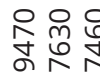

总点䍃导导

总

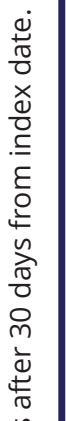

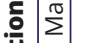

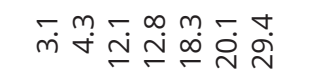

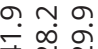

N $-m \circ-$

ลั่

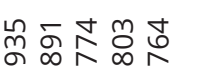

옫욛용

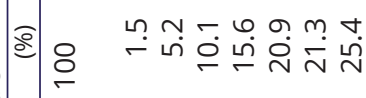

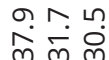

ก $\infty$.

ล่ำ

迎

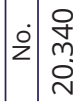

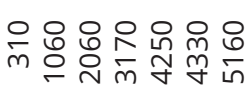

욧ㅇํㅇ

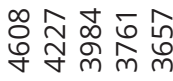

\section{。}

은 는

$\sqrt{2}$

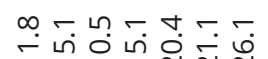

$\dot{\infty} \div-\dot{m}$

ㅇ. $0 .-\pi+4$

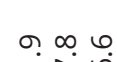

প্লে :

这

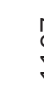

ลิ下这

のุ

in

হু

กั

㞫命

ㄴ.

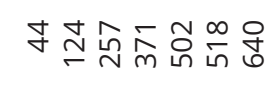

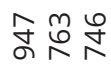

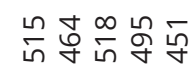

쏘ำ 욤

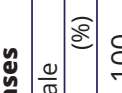

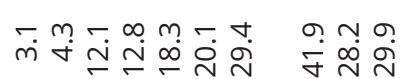

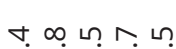

$\operatorname{sed}$

กู่ बู่

$\dot{\sim}+\stackrel{\infty}{\circ} \dot{\sim}$

๖ ำ นก

$\dot{2} \mid \stackrel{\text { ช }}{ }$

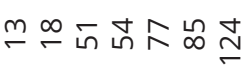

$\stackrel{ }{ } \stackrel{\circ}{\rightleftharpoons}$

ำกำ

$\stackrel{\infty}{m} \stackrel{\sim}{n}$

๑

은

กำ ำ

ำ. …ำ

m.

๑ุ

ம்

守市

$\stackrel{\llcorner}{\leftarrow}$

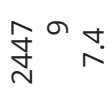

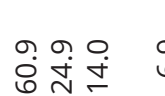

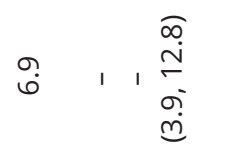

๗ั山

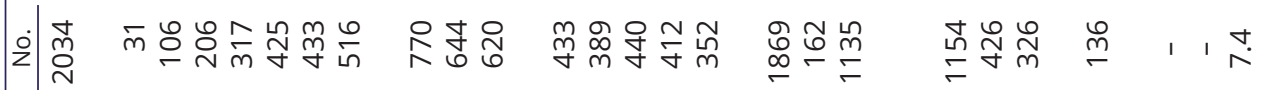
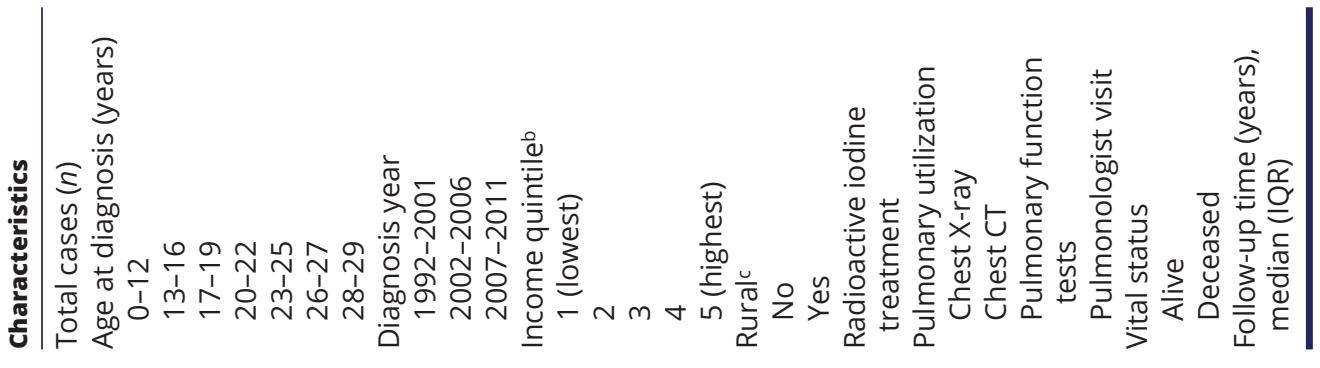

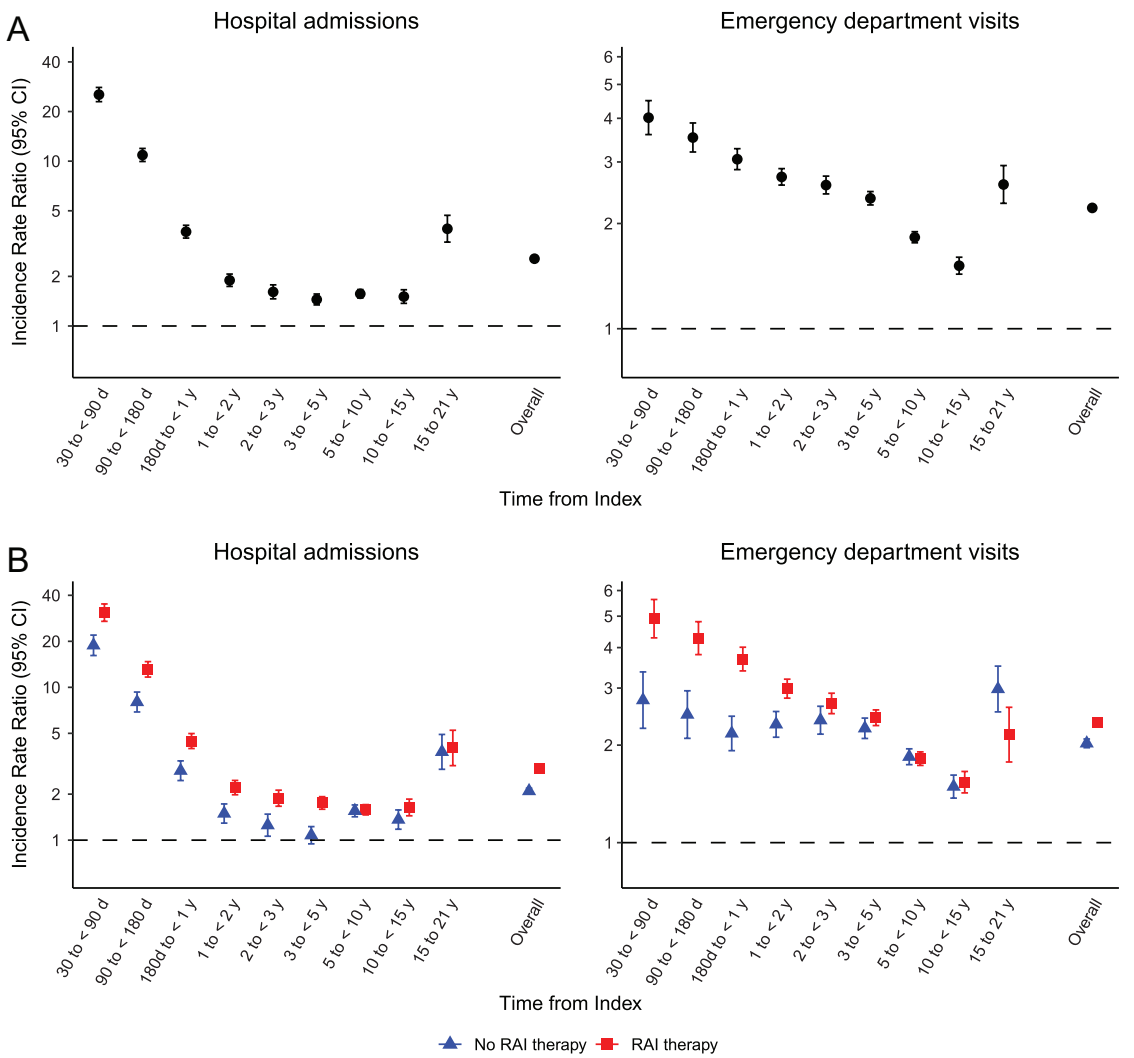

\begin{abstract}
Figure 1
All-cause hospitalization and emergency department visits for primary differentiated thyroid carcinoma patients. (A) Overall and (B) stratified by radioactive iodine therapy. $d$, days; $y$, years; RAl, radioactive iodine therapy. A full colour version of this figure is available at https://doi. org/10.1530/ERC-19-0463.
\end{abstract}

CT for DTC patients and controls were 51.7 and 3.0 per 1000 person-years, respectively, representing an IRR of 17.5 (95\% CI, 15.8-19.3). PFT had IRR 1.8 (95\% CI, 1.7-1.9) for DTC cases compared to controls (Table 2). Rates of CXR and chest CT were highest in the 30- to 90-day time-period following the index date and decreased over time (Fig. 2A). An increase in IRR was observed in the 15- to 21-year time-period for all HSU when compared to the preceding 10 to 15 -year time period. Patients who underwent RAI treatment accessed pulmonary-related health resources more often than those who did not (Fig. 2B). Absolute incidence rates for pulmonary-related HSU are presented in Supplementary Table 4.

To ensure that any observed changes in pulmonaryrelated HSU did not reflect an artifact of the administrative data, a 'negative tracer' analysis examining the utilization of a diagnostic modality unlikely to be associated with DTC or its treatment, abdominal/pelvic CT, was undertaken to compare to chest imaging (Supplementary Fig. 1).

\section{Outpatient physician visits}

The distribution of IRRs for physician billing records varied between subspecialty disciples (Fig. 3). Not surprisingly, visits to Endocrinologists and Otolaryngologists demonstrated the highest relative incidence among disciplines for DTC patients compared to controls with an overall IRR of 43.48 (95\% CI, 40.34-46.87) and 10.89 (95\% CI, 10.57-11.22), respectively. Overall, the IRR of respiratory disease specialist billings was 2.25 (95\% CI, 2.06-2.44). There was a relative excess of visits to pulmonologists among DTC patients versus controls in all periods of observation except during the 2 to 3 years (IRR, 1.16; 95\% CI, 0.82-1.65) and 3 to 5 years (IRR, 1.12; 95\% CI, 0.87-1.46) after the index date. A relative increase in visits to pulmonologists was observed beginning five years after diagnosis and thereafter. Figure 3 illustrates a rise in the IRRs of all subspecialty billings in the 15- to 21-year time period, concordant with the increases seen with diagnostic imaging of the chest and PFTs. The absolute incidence rates for outpatient physician visits is presented in Supplementary Table 5.

\section{Discussion}

In this population-based retrospective study, we describe increased rates of all-cause hospital admissions, ED visits, outpatient physician (generalist and specialist)
(C) 2020 Society for Endocrinology Published by Bioscientifica Ltd. Printed in Great Britain 

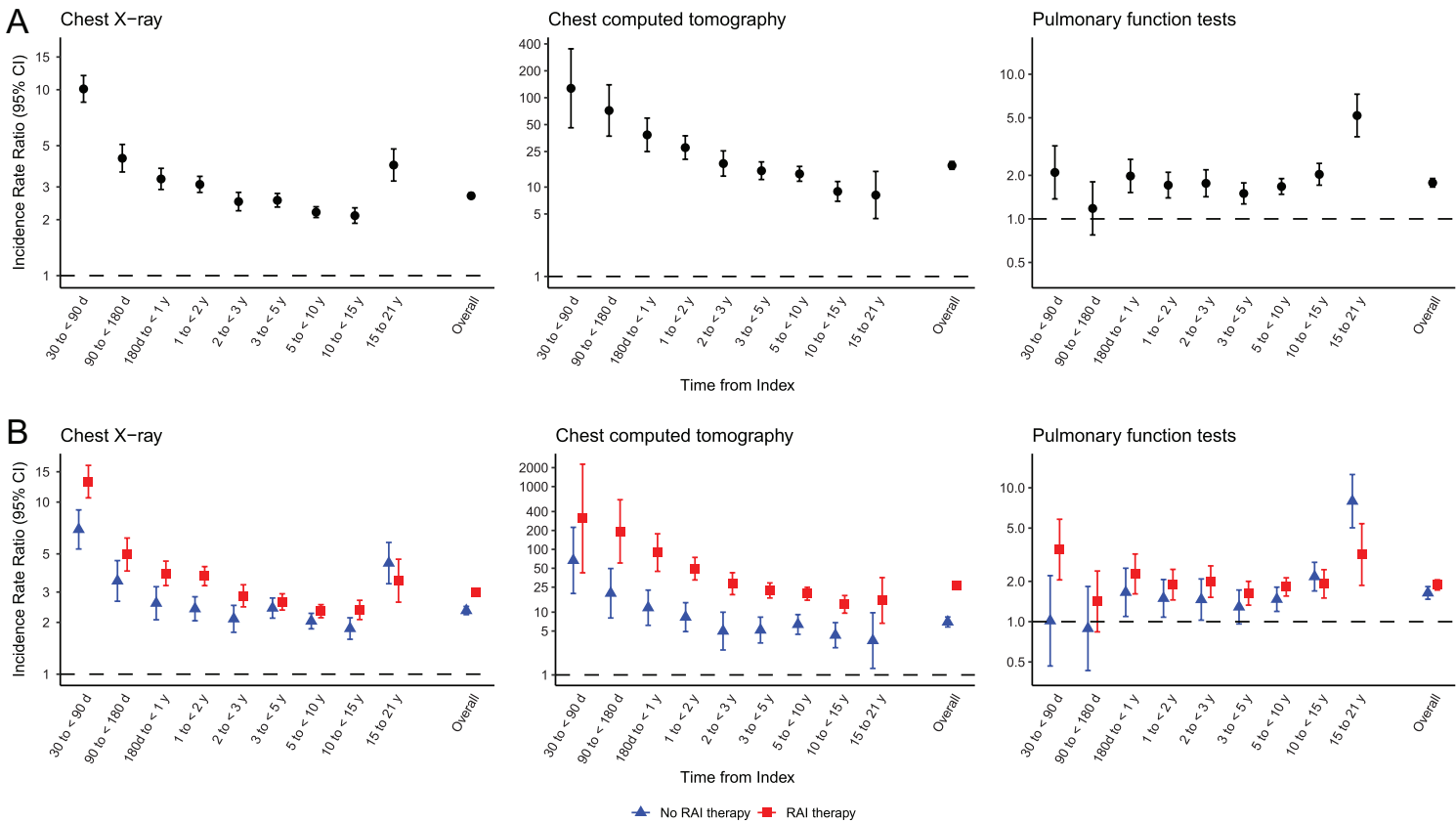

Figure 2

Incidence rate ratio of pulmonary specific healthcare services utilization for primary differentiated thyroid cancer patients. (A) Overall and (B) stratified by radioactive iodine therapy. d, days; y, years; RAl, radioactive iodine therapy. A full colour version of this figure is available at https://doi.org/10.1530/ ERC-19-0463.

visits, and pulmonary-related diagnostic testing, among DTC patients during the first 21 years of survivorship compared to matched population controls. Given the generally favorable prognosis of CAYA DTC, these results were somewhat unanticipated. Temporal trends in the incidence rate utilization of healthcare services were noted over the entire follow-up period, with the largest IRR in the period immediately following the diagnosis of DTC, decaying as time from treatment elapsed. These findings are not unexpected, as the most intense interaction with the health system would be expected in close temporal proximity to initial treatment of DTC with tapering utilization thereafter. We did, however, observe a sustained excess utilization of all modalities of health service late into survivorship, long after a period wherein such utilization would be anticipated as part of routine surveillance. In contrast, usage of abdominal/ pelvic CT demonstrated no clear trend over time, rather IRR fluctuated between two and four over the entire duration of follow-up (Supplementary Fig. 1).

Survivors of CAYA DTC demonstrated an overall IRR of CXR and pulmonary CT utilization of 2.7 (95\% CI, 2.6-2.8) and 17.5 (95\% CI, 15.8-19.3) times greater, respectively, than matched population controls who did not experience cancer. Heightened utilization of diagnostic medical imaging seen in our study align with other studies in young adults which have defined patterns of diagnostic imaging in long-term survivors of AYA cancer (Daly et al. 2015). A recent study by Daly and colleagues (Daly et al. 2015) report the diagnostic imaging rates in long-term survivors of thyroid carcinoma were 1.32 (95\% CI, 1.26-1.38) and 1.93 (95\% CI, 1.73-2.16) times higher for CXR and chest CT, respectively, among AYAs aged 20-44 years compared to cancer-free controls after 5-15 years post diagnosis. While our study focused exclusively on pulmonary-related diagnostic imaging in CAYAs (aged 29 and younger), both studies demonstrate that individuals who experience thyroid cancer early in life interact with the healthcare system more frequently than the general population, which persists long into survivorship.

Importantly, while these data identify increased utilization of the healthcare system by DTC survivors, they do not specifically identify increased morbidity. In considering diagnostic testing (i.e. imaging, PFTs), we cannot exclude a high rate of testing that is not justified by underlying disease. Further exploration of this may identify an opportunity for significant cost-savings by reduction of unnecessary testing through clinician and patient education. Alternatively, it may identify a reservoir of pulmonary disease that has thus far gone undescribed. Of late, the principal of 'Choosing Wisely' 


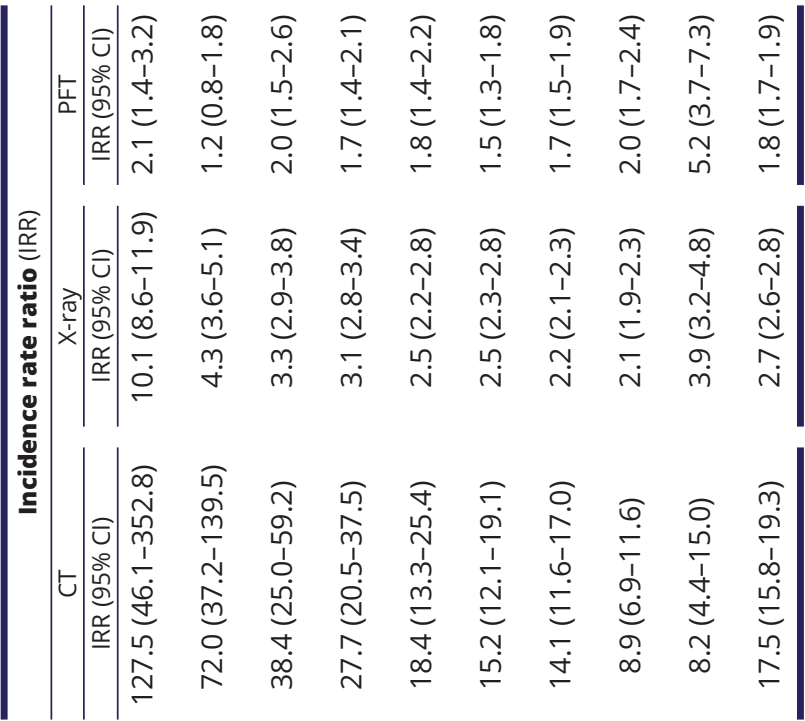

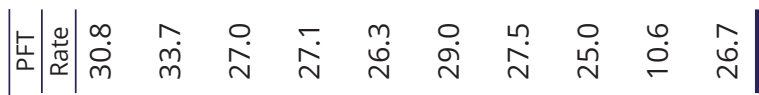

离|

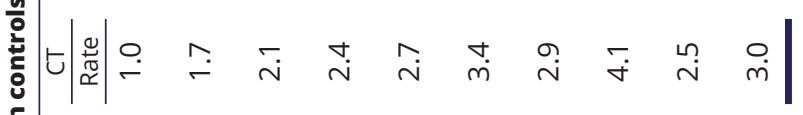

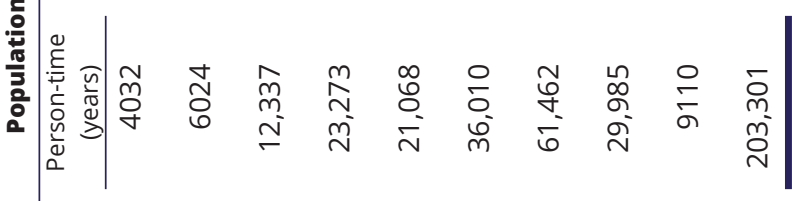

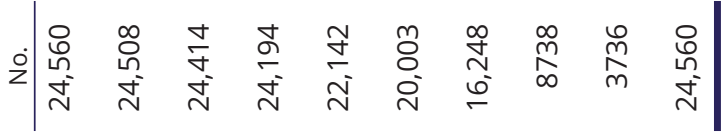

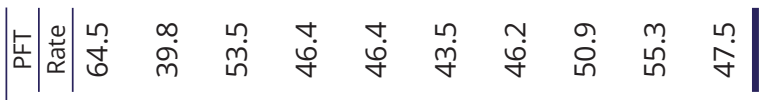

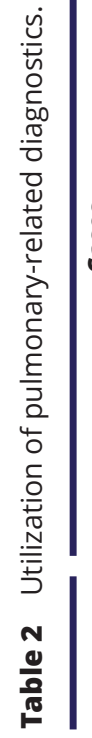

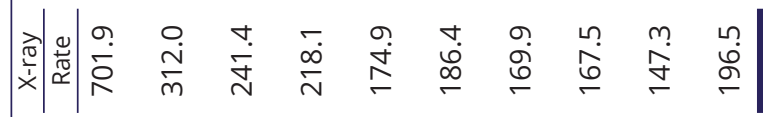

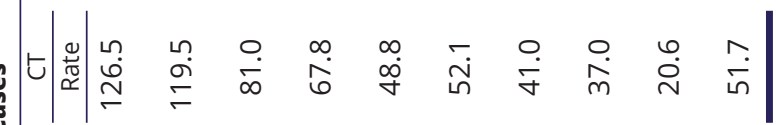

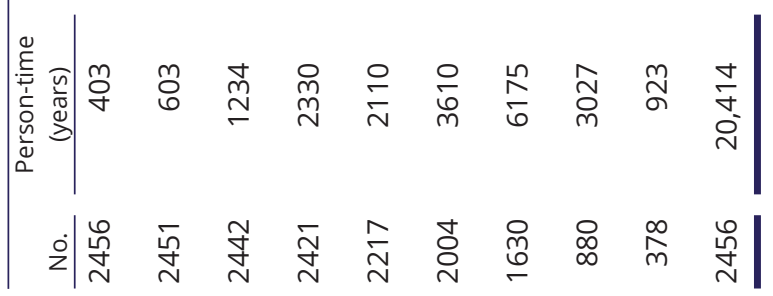

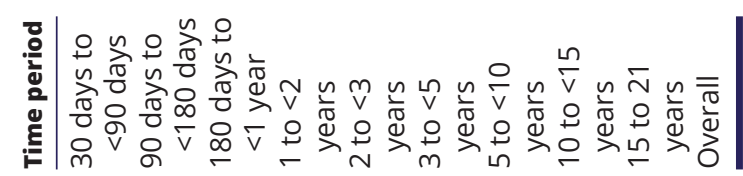




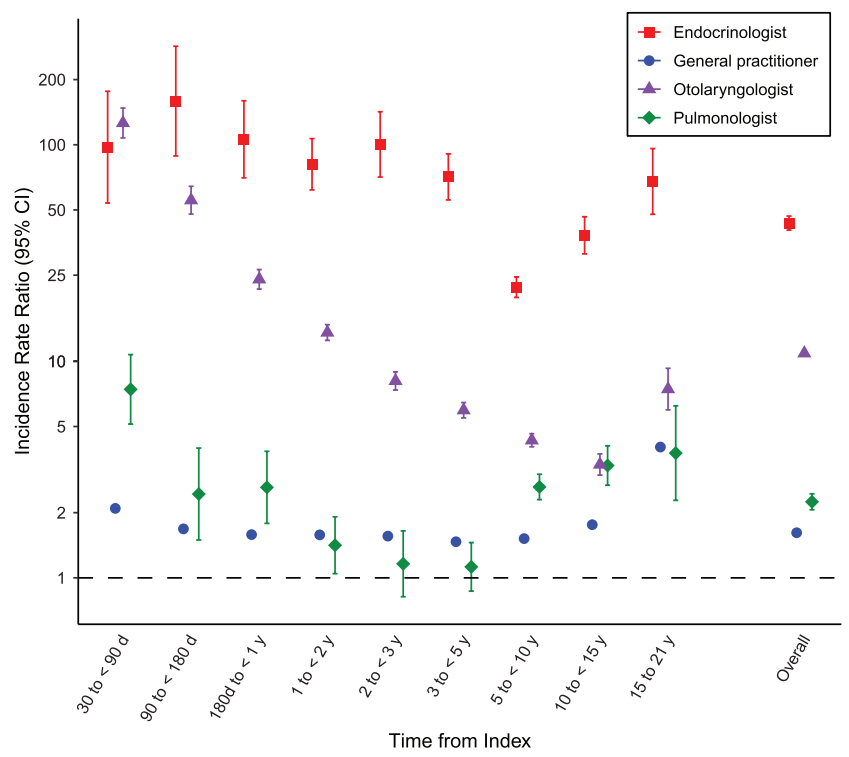

Figure 3

Fee-for-service physician billing for primary differentiated thyroid cancer patients. $d$, days; $y$, years. A full colour version of this figure is available at https://doi.org/10.1530/ERC-19-0463.

has challenged conventional medical practice, advocating clinicians to perform only necessary diagnostics in an effort to reduce healthcare overuse (Cassel \& Guest 2012, Coon et al. 2019, Morgan et al. 2019). Paradigm shifts eliminating CT scans in other favorable-outcome pediatric cancer surveillance programs have demonstrated that survival is not compromised, while affording a reduction in radiation exposure and associated healthcare costs (Mullen et al. 2018); such efforts may therefore apply to the DTC population as well.

While a true excess of medical complexity is likely among survivors, other explanations for increased access to the healthcare system also likely contribute to the observed excess. First, lifelong surveillance for tumor recurrence is advocated, generating an obligate, albeit small, increase in HSU. In addition, most survivors in this cohort underwent total thyroidectomy and require routine monitoring of thyroid replacement in the context of visit to primary-care physician or specialists. Additionally, we speculate that there is likely a referral bias where primary care providers' identification of patients as cancer survivors may prompt a lower threshold to investigate or refer medical complaints that may not have been otherwise referred, absent a cancer history.

One must also consider survivors' perceptions of medical vulnerability and its contribution to increased HSU (Morley \& Goldfarb 2015, Goldfarb \& Casillas 2016). Evron and colleagues recently demonstrated differences in rates of physician visits, ultrasounds and RAI scans among DTC survivors based on an individuals' propensity to 'minimize' vs 'maximize' health risk in the post-operative phase (Evron et al. 2019). AYAs experience more fear of recurrence than adult survivors of cancer in general, with up to 85\% of AYA survivors expressing recurrence fear (Yang et al. 2019). Cancer-related worry has also been described among AYA survivors of DTC (Bresner et al. 2015). This cancer-related worry and fear of cancer recurrence translates into increased healthcare utilization in survivors (Lebel et al. 2013). Indeed, healthseeking behaviors among DTC survivors may contribute to the excess utilization observed in the current study (Evron et al. 2019).

Substantial differences in HSU were observed between RAI and non-RAI treated patients in the early time periods after diagnosis. While previous studies of RAI therapy support an increased rate of HSU early in treatment (Haymart et al. 2011), it does not fully explain the heightened utilization we observed in individuals who did not undergo RAI therapy, nor can it explain the sustained excess utilization $5+$ years into survivorship.

Patients in this cohort who underwent RAI therapy had increased rates of pulmonary imaging and a higher incidence of hospitalizations and ED visits. A proportion of this group will have had distal (primarily pulmonary) metastatic disease, which would justify ongoing thoracic imaging. However, the $56.6 \%$ of patients who underwent RAI in this cohort far exceeds the estimated $7.6-27 \%$ (Hogan et al. 2009, Golpanian et al. 2016) with pulmonary metastases. Thus, by inference, a majority of patients treated with RAI had M0 disease.

RAI therapy is associated with an increased risk of secondary malignancy, including that of the hematopoietic system, gastrointestinal tract and salivary glands (Sawka etal. 2009). In this cohort, we previously reported a total of 17 patients with secondary malignancies (Pole et al. 2017). This low incidence is therefore unlikely to have contributed substantially to healthcare utilization in the current analysis. Pulmonary fibrosis may develop as a consequence of RAI treatment of patients with diffuse iodine-avid pulmonary metastases, particularly after multiple courses and/or high cumulative exposure (Albano et al. 2017). Changes in PFTs have been associated with RAI therapy and pulmonary metastases of DTC (Jang et al. 2015). Thus, a causal link between increased pulmonary diagnostic testing and respiratory disease is plausible, albeit not demonstrated in the current study.

A common observation in this DTC survivor cohort was a decaying rate of HSU as time from diagnosis 
elapsed, with a noticeable upward inflection in usage of all modalities of in the period 15-21 years post diagnosis. Negative-tracer results from abdominal CT scans did not mimic this trend (Supplementary Fig. 1). Pregnancy and childbirth, genitourinary diseases, and digestive system diseases were identified as main reasons for hospital admission, and there were no differences in the diagnostic reasons in later years of our study between DTC patients and population controls (Supplementary Table 3). Late into survivorship, DTC patients were hospitalized at a higher rate than matched population controls, although indications for admission are comparable (Fig. 2 and Supplementary Table 3). While we report IRRs for HSU relative to matched controls, absolute incidence rates for all HSU and pulmonary-related utilization presented in Supplementary Tables 2, 4 and 5 illustrate that the incidence of HSU for controls was consistent throughout the study period, whereas among DTC cases it remained higher.

This study benefits from the use of a provincially mandated cancer registry, permitting population capture of thyroid carcinomas diagnosed between 1992 and 2011. We were also able to capture all healthcare system interactions of individuals in our cohort using physician billing records and administrative databases in our singlepayer healthcare system. This allowed the comprehensive monitoring of HSU among affected patients from the time of diagnosis date until the end of study.

While this matched cohort study design robustly controls for baseline confounding by normalizing against a control population, several limitations must be considered when interpreting the results of this study. First, the use of population-based administrative databases allowed the assessment of a large CAYA DTC cohort longitudinally at the expense of detailed individual-level data. Second, the clinical care of DTC evolved over the 21-year period of study with varying approaches to treatment and posttreatment surveillance. Third, this population-level data set does not capture individual cancer staging, precluding an association between stage and utilization, nor can these data associate individual therapeutic exposures such as RAI (or administered activity) with subsequent health or HSU outcomes. Finally, response to therapy and/or relapses in disease could not be determined and limited our ability to control for advanced disease, which may have confounded healthcare utilization analysis.

Together, these observations identify that survivors of CAYA DTC interact more frequently with the healthcare system than matched population controls. This is true of ED visits, hospital admissions and outpatient physician visits. Survivors also undergo more frequent thoracic imaging, suggesting the possibility of excess pulmonary risk, although this is speculative at this point. Not surprisingly, access to the healthcare system is greatest immediately after diagnosis and decays over time, yet utilization never returns to levels observed in population controls. Cross-sectional and/or prospective longitudinal studies of survivors and their medical needs will ultimately be required to provide a better resolution of this question; however, this study raises the possibility of excess morbidity among survivors of CAYA DTC.

We cannot explain the late inflection in usage, however, rigorous re-examination of the primary data and comparison to other modes of healthcare utilization suggest it to be a genuine signal. Future research should explore the long-term adverse events of DTC therapy in children and AYAs, 15+ years into survivorship, which could further identify late sequelae and healthcare system use. Furthermore, studies should explore the economic impact of DTC survivorship on the healthcare system, and identify opportunities for optimization of resource utilization.

\section{Supplementary materials}

This is linked to the online version of the paper at https://doi.org/10.1530/ ERC-19-0463.

\section{Declaration of interest}

The authors declare that there is no conflict of interest that could be perceived as prejudicing the impartiality of the research reported.

\section{Funding}

Funding for this project was provided by a peer-reviewed Seed Grant from the Pediatric Oncology Group of Ontario.

\section{Author contribution statement}

G D, J D W, and J D P conceived and designed the study. G D and A M Z procured the study data set and performed the data analysis. J D P and J D W supervised data analyses. G D wrote the first draft of the manuscript and all authors contributed to subsequent drafts and approved the manuscript before submission for publication. All authors had full access to the study data and vouch for the integrity and accuracy of the analysis. J D W and J D $\mathrm{P}$ : Co-senior authors.

\section{Acknowledgements}

Funding for this project was provided by a peer-reviewed Seed Grant from the Pediatric Oncology Group of Ontario. This study was supported through provision of data by ICES and Cancer Care Ontario (CCO), and through funding support to ICES from an annual grant by the Ministry of Health and Long-Term Care (MOHLTC) and the Ontario Institute for Cancer Research (OICR). The opinions, results and conclusions reported in this paper are those of the authors. No endorsement by i.e. CCO, OICR or the Government of Ontario is intended or should be inferred.
(C) 2020 Society for Endocrinology Published by Bioscientifica Ltd. Printed in Great Britain 


\section{References}

Albano D, Bertagna F, Panarotto MB \& Giubbini R 2017 Early and late adverse effects of radioiodine for pediatric differentiated thyroid cancer. Pediatric Blood and Cancer 64 e26595. (https://doi. org/10.1002/pbc.26595)

Alzahrani AS, Alswailem M, Moria Y, Almutairi R, Alotaibi M, Murugan AK, Qasem E, Alghamdi B \& Al-Hindi H 2019 Lung metastasis in pediatric thyroid cancer: radiological pattern, molecular genetics, response to therapy, and outcome. Journal of Clinical Endocrinology and Metabolism 104 103-110. (https://doi.org/10.1210/ jc.2018-01690)

Araque DVP, Bleyer A \& Brito JP 2017 Thyroid cancer in adolescents and young adults. Future Oncology 13 1253-1261. (https://doi. org/10.2217/fon-2017-0024)

Bal CS, Kumar A, Chandra P, Dwivedi SN \& Mukhopadhyaya S 2004 Is chest $\mathrm{x}$-ray or high-resolution computed tomography scan of the chest sufficient investigation to detect pulmonary metastasis in pediatric differentiated thyroid cancer? Thyroid 14 217-225. (https:// doi.org/10.1089/105072504773297894)

Bergstralh EJ, Kosanke JL \& Jacobsen SJ 1996 Software for optimal matching in observational studies. Epidemiology 7 331-332.

Bresner L, Banach R, Rodin G, Thabane L, Ezzat S \& Sawka AM 2015 Cancer-related worry in Canadian thyroid cancer survivors. Journal of Clinical Endocrinology and Metabolism 100 977-985. (https://doi. org/10.1210/jc.2014-3169)

Canadian Institute for Health Information 2012a International Statistical Classification of Diseases and Related Health Problems, Tenth Revision, Canada (ICD-10-CA), Volume 1 - Tabular List. Ottawa, Canada: Canadian Institute for Health Information.

Canadian Institute for Health Information $2012 b$ Canadian Coding Standards for Version 2012 ICD-10-CA and CCI. Ottawa, Canada: Canadian Institute for Health Information.

Cassel CK \& Guest JA 2012 Choosing wisely: helping physicians and patients make smart decisions about their care. JAMA $\mathbf{3 0 7}$ 1801-1802. (https://doi.org/10.1001/jama.2012.476)

Chopra S, Garg A, Ballal S \& Bal CS 2015 Lung metastases from differentiated thyroid carcinoma: prognostic factors related to remission and disease-free survival. Clinical Endocrinology $\mathbf{8 2}$ 445-452. (https://doi.org/10.1111/cen.12558)

Coon ER, Quinonez RA, Morgan DJ, Dhruva SS, Ho T, Money N \& Schroeder AR 20192018 update on pediatric medical overuse: a review. JAMA Pediatrics 173 379-384. (https://doi.org/10.1001/ jamapediatrics.2018.5550)

Daly C, Urbach DR, Stukel TA, Nathan PC, Deitel W, Paszat LF, Wilton AS \& Baxter NN 2015 Patterns of diagnostic imaging and associated radiation exposure among long-term survivors of young adult cancer: a population-based cohort study. BMC Cancer 15612. (https://doi.org/10.1186/s12885-015-1578-1)

Durante C, Haddy N, Baudin E, Leboulleux S, Hartl D, Travagli JP, Caillou B, Ricard M, Lumbroso JD, De Vathaire F, et al. 2006 Longterm outcome of 444 patients with distant metastases from papillary and follicular thyroid carcinoma: benefits and limits of radioiodine therapy. Journal of Clinical Endocrinology and Metabolism 91 2892-2899. (https://doi.org/10.1210/jc.2005-2838)

Earle CC 2014 Ontario cancer data linkage project: 'cd-link'. Healthcare Quarterly 17 11-13. (https://doi.org/10.12927/hcq.2014.23786)

Evron JM, Reyes-Gastelum D, Banerjee M, Scherer LD, Wallner LP, Hamilton AS, Ward KC, Hawley ST, Zikmund-Fisher BJ \& Haymart MR 2019 Role of patient maximizing-minimizing preferences in thyroid cancer surveillance. Journal of Clinical Oncology 37 3042-3049. (https://doi.org/10.1200/JCO.19.01411)

Francis GL, Waguespack SG, Bauer AJ, Angelos P, Benvenga S, Cerutti JM, Dinauer CA, Hamilton J, Hay ID, Luster M, et al. 2015 Management guidelines for children with thyroid nodules and differentiated thyroid cancer. Thyroid 25 716-759. (https://doi. org/10.1089/thy.2014.0460)

Gershon AS, Victor JC, Guan J, Aaron SD \& To T 2012 Pulmonary function testing in the diagnosis of asthma: a population study. Chest 141 1190-1196. (https://doi.org/10.1378/chest.11-0831)

Goldfarb M \& Casillas J 2016 Thyroid cancer-specific quality of life and health-related quality of life in young adult thyroid cancer survivors. Thyroid 26 923-932. (https://doi.org/10.1089/ thy.2015.0589)

Golpanian S, Perez EA, Tashiro J, Lew JI, Sola JE \& Hogan AR 2016 Pediatric papillary thyroid carcinoma: outcomes and survival predictors in 2504 surgical patients. Pediatric Surgery International 32 201-208. (https://doi.org/10.1007/s00383-015-3855-0)

Haymart MR, Banerjee M, Stewart AK, Koenig RJ, Birkmeyer JD \& Griggs JJ 2011 Use of radioactive iodine for thyroid cancer. JAMA 306 721-728. (https://doi.org/10.1001/jama.2011.1139)

Hogan AR, Zhuge Y, Perez EA, Koniaris LG, Lew JI \& Sola JE 2009 Pediatric thyroid carcinoma: incidence and outcomes in 1753 patients. Journal of Surgical Research 156 167-172. (https://doi. org/10.1016/j.jss.2009.03.098)

Jang EK, Kim WG, Kim HC, Huh JW, Kwon H, Choi YM, Jeon MJ, Kim TY, Shong YK, Ryu JS, et al. 2015 Changes in the pulmonary function test after radioactive iodine treatment in patients with pulmonary metastases of differentiated thyroid cancer. PLOS ONE 10 e0125114. (https://doi.org/10.1371/journal.pone.0125114)

Kloos RT, Eng C, Evans DB, Francis GL, Gagel RF, Gharib H, Moley JF, Pacini F, Ringel MD, et al. 2009 Medullary thyroid cancer: management guidelines of the American Thyroid Association. Thyroid 19 565-612. (https://doi.org/10.1089/thy.2008.0403)

Lazar L, Lebenthal Y, Segal K, Steinmetz A, Strenov Y, Cohen M, Yaniv I, Yackobovitch-Gavan M \& Phillip M 2016 Pediatric thyroid cancer: postoperative classifications and response to initial therapy as prognostic factors. Journal of Clinical Endocrinology and Metabolism 101 1970-1979. (https://doi.org/10.1210/jc.2015-3960)

Lebel S, Tomei C, Feldstain A, Beattie S \& McCallum M 2013 Does fear of cancer recurrence predict cancer survivors' health care use? Supportive Care in Cancer 21 901-906. (https://doi.org/10.1007/ s00520-012-1685-3)

Lim H, Devesa SS, Sosa JA, Check D \& Kitahara CM 2017 Trends in thyroid cancer incidence and mortality in the United States, 19742013. JAMA 317 1338-1348. (https://doi.org/10.1001/ jama.2017.2719)

Livhits MJ, Pasternak JD, Xiong M, Li N, Gosnell JE, Yeh MW \& Chiu HK 2016 Pre-ablation thyroglobulin and thyroglobulin to thyroid-stimulating hormone ratio may be associated with pulmonary metastases in children with differentiated thyroid cancer. Endocrine Practice 22 1259-1266. (https://doi.org/10.4158/EP161360. OR)

Marti JL, Jain KS \& Morris LG 2015 Increased risk of second primary malignancy in pediatric and young adult patients treated with radioactive iodine for differentiated thyroid cancer. Thyroid $\mathbf{2 5}$ 681-687. (https://doi.org/10.1089/thy.2015.0067)

Massimino M, Evans DB, Podda M, Spinelli C, Collini P, Pizzi N \& Bleyer A 2018 Thyroid cancer in adolescents and young adults. Pediatric Blood and Cancer 65 e27025. (https://doi.org/10.1002/pbc.27025)

Morgan DJ, Dhruva SS, Coon ER, Wright SM \& Korenstein D 2019 2019 update on medical overuse: a review. JAMA Internal Medicine 179 1568-1574. (https://doi.org/10.1001/ jamainternmed.2019.3842)

Morley S \& Goldfarb M 2015 Support needs and survivorship concerns of thyroid cancer patients. Thyroid 25 649-656. (https://doi. org/10.1089/thy.2015.0032)

Mullen EA, Chi YY, Hibbitts E, Anderson JR, Steacy KJ, Geller JI, Green DM, Khanna G, Malogolowkin MH, Grundy PE, et al. 2018 Impact of surveillance imaging modality on survival after recurrence in patients with favorable-histology wilms tumor: a report from the https://erc.bioscientifica.com

https://doi.org/10.1530/ERC-19-0463 (c) 2020 Society for Endocrinology Published by Bioscientifica Ltd. Printed in Great Britain 
Children's Oncology Group. Journal of Clinical Oncology 36 JCO1800076. (https://doi.org/10.1200/JCO.18.00076)

Ontario Ministry of Health and Long Term Care 2015 Schedule of Benefits: Physician Services under the Health Insurance Act. Toronto, Canada: Ontario Ministry of Health and Long Term Care.

Pawelczak M, David R, Franklin B, Kessler M, Lam L \& Shah B 2010 Outcomes of children and adolescents with well-differentiated thyroid carcinoma and pulmonary metastases following ${ }^{131} \mathrm{I}$ treatment: a systematic review. Thyroid 20 1095-1101. (https://doi. org/10.1089/thy.2009.0446)

Pole JD, Zuk AM \& Wasserman JD 2017 Diagnostic and treatment patterns among children, adolescents, and young adults with thyroid cancer in Ontario: 1992-2010. Thyroid 27 1025-1033. (https://doi.org/10.1089/thy.2016.0629)

Sawka AM, Thabane L, Parlea L, Ibrahim-Zada I, Tsang RW, Brierley JD, Straus S, Ezzat S \& Goldstein DP 2009 Second primary malignancy risk after radioactive iodine treatment for thyroid cancer: a systematic review and meta-analysis. Thyroid 19 451-457. (https:// doi.org/10.1089/thy.2008.0392)

Song HJ, Qiu ZL, Shen CT, Wei WJ \& Luo QY 2015 Pulmonary metastases in differentiated thyroid cancer: efficacy of radioiodine therapy and prognostic factors. European Journal of Endocrinology $\mathbf{1 7 3}$ 399-408. (https://doi.org/10.1530/EJE-15-0296)
Statistics Canada 1986 Canadian Classification of Diagnostic, Therapeutic and Surgical Procedures. Ottawa, Canada: Statistics Canada, Health Division, Nosology Reference Centre.

US Department of Health and Human Services 1988 International Classification of Diseases, 9th Revision (Clinical Modification). Atlanta, GA, USA: Centers for Disease Control and Prevention.

Vergamini LB, Frazier AL, Abrantes FL, Ribeiro KB \& RodriguezGalindo C 2014 Increase in the incidence of differentiated thyroid carcinoma in children, adolescents, and young adults: a populationbased study. Journal of Pediatrics 164 1481-1485. (https://doi. org/10.1016/j.jpeds.2014.01.059)

World Health Organization 2013 International Classification of Diseases for Oncology: ICD-O, 3rd ed., first revision. Eds A Fritz, C Percy, A Jack, K Shanmugaratnam, L Sobin, D Parkin \& S Whelan. Geneva, Switzerland: World Health Organization.

Yang Y, Li W, Wen Y, Wang H, Sun H, Liang W, Zhang B \& Humphris G 2019 Fear of cancer recurrence in adolescent and young adult cancer survivors: a systematic review of the literature. Psycho-Oncology $\mathbf{2 8}$ 675-686. (https://doi.org/10.1002/pon.5013)

Zhang Y, Liang J, Li H, Cong H \& Lin Y 2016 Risk of second primary breast cancer after radioactive iodine treatment in thyroid cancer: a systematic review and meta-analysis. Nuclear Medicine Communications 37 110-115. (https://doi.org/10.1097/MNM.000000000000419)

Received in final form 17 March 2020

Accepted 5 April 2020

Accepted Manuscript published online 6 April 2020 (c) 2020 Society for Endocrinology Published by Bioscientifica Ltd. Printed in Great Britain 